\title{
Age-Related Decrease in Inferior Vena Cava Diameter Measured with Echocardiography
}

\author{
Hisashi Masugata, ${ }^{1}$ Shoichi Senda, ${ }^{1}$ Hiroyuki Okuyama, ${ }^{1}$ Koji Murao, ${ }^{2}$ \\ Michio Inukai, ${ }^{1}$ Naohisa Hosomi, ${ }^{3}$ Yasuyoshi Iwado, ${ }^{4}$ Takahisa Noma, ${ }^{4}$ \\ Masakazu Kohno, ${ }^{4}$ Takashi Himoto ${ }^{1}$ and Fuminori Goda ${ }^{1}$

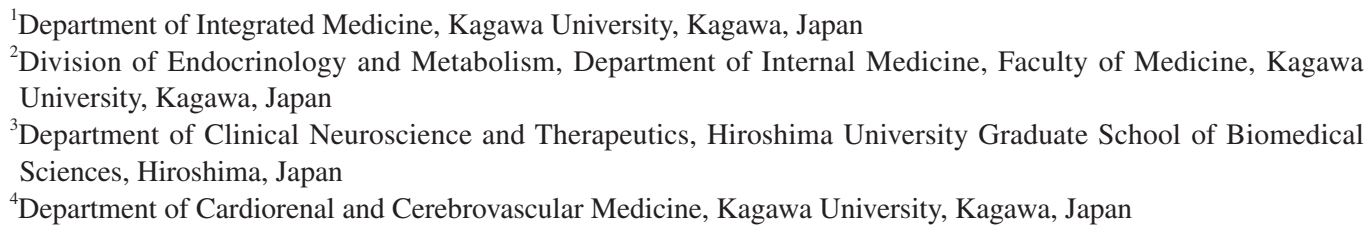

The diameter of the inferior vena cava (IVC) measured with echocardiography is clinically used as a parameter to estimate right atrial pressure, which reflects dehydration or overhydration. Because elderly patients fall easily into dehydration, normal values for IVC diameters in elderly patients may be helpful for geriatric medicine. However, normal values of IVC diameter in relation to age have not been investigated. The purpose of this study was to elucidate age-related changes in IVC diameter using echocardiography. Enrolled in the study were 200 patients (67 \pm 15 yrs: range 17-94 yrs) with cardiovascular risk factors but no overt cardiac diseases. IVC diameters throughout the respiratory cycle were measured as maximum and minimum IVC diameters (IVC $\max _{\text {, }}, \mathrm{IVC}_{\min }$ ) using M-mode echocardiography. To assess IVC collapsibility, the respirophasic variation of IVC diameter was calculated as $\left(I V C_{\max }-I V C_{\min }\right) /\left(I V C_{\max }\right) \times 100$. Maximum IVC diameter was decreased with advancing age $(r=-0.221, p=0.002)$. The respirophasic variation of the IVC diameter was increased with advancing age $(r=0.244, p=0.001)$. Stepwise multiple regression analysis showed that age was an independent determinant for both maximum IVC diameter $(B$ coefficient $=$ $-0.249, p<0.001$ ) and respirophasic variation of the IVC diameter ( $B$ coefficient $=0.268, p<0.001$ ). Age-related decrease in maximum IVC diameter and increase in the respirophasic IVC collapsibility may indicate the decrease in right atrial pressure in some elderly patients. Therefore, elderly patients with decreased maximum IVC and increased respirophasic IVC collapsibility may need prevention for dehydration.

Keywords: inferior vena cava diameter; echocardiography; cardiac preload; aging

Tohoku J. Exp. Med., 2010, 222 (2), 141-147. (C) 2010 Tohoku University Medical Press

Several echocardiographic parameters can provide information regarding blood volume retention or dehydration. The diameter of the inferior vena cava (IVC) measured with echocardiography is clinically used as a parameter to estimate right atrial pressure, which reflects dehydration or overhydration. It is important to evaluate hydration status in the clinical settings in elderly people, who fall easily into dehydration. Especially for elderly patients with dementia, it is difficult to complain their symptoms of dehydration. Therefore, normal values for IVC diameters in elderly patients may be helpful for geriatric medicine. However, normal values of IVC diameter in relation to age have not been investigated. Some previous studies (Watanabe et al. 2005; Daimon et al. 2008) demonstrated the normal values for echocardiographic measure- ments and evaluated the relationship between these parameters and age in a large healthy Japanese population. However, these studies did not include data regarding IVC diameters. Although some previous studies (Simonson and Schiller 1988; Kircher et al. 1990; Nagueh et al. 1996; Brennan et al. 2007) demonstrated normal values of IVC diameters for estimating right atrial pressure, these previous studies were not performed in the Japanese population. Therefore, there are no data regarding normal values of the IVC diameter in relation to age in the Japanese population. In this study, we investigated age-related changes in IVC diameter measured with echocardiography in Japanese patients without overt cardiac disease.

Received August 12, 2010; revision accepted for publication September 21, 2010. doi: 10.1620/tjem.222.141

Correspondence: Hisashi Masugata, M.D., Ph.D., Department of Integrated Medicine, Kagawa University, 1750-1, Miki-cho, Kita-gun, Kagawa 761-0793, Japan.

e-mail: masugata@med.kagawa-u.ac.jp 


\section{Methods}

\section{Subjects and Protocol}

The study subjects were 200 consecutive patients (112 male, 88 female; mean age $67 \pm 15$ years, range 17-94 years) who were diagnosed as having cardiovascular risk factors. The cardiovascular risk factors included in this study were hypertension, diabetes mellitus, and dyslipidemia, which were respectively diagnosed according to the guidelines of the Japanese Society of Hypertension, the Japan Diabetes Society and the Japan Atherosclerosis Society. Patients with a history of heart failure or obvious heart disease were excluded. None of the subjects had a history of atherosclerotic cardiovascular disease or stroke. Patients with atrial fibrillation were excluded. Although patients with mild tricuspid valve regurgitation were included to this study, those with moderate or severe tricuspid regurgitation were excluded. Blood pressure was determined using the conventional cuff method at the time when the echocardiographic examination was performed. Hypertension was defined as systolic blood pressure $\geq 140 \mathrm{mmHg}$ and/or diastolic blood pressure $\geq 90 \mathrm{mmHg}$. Echocardiographic examinations were performed to assess cardiac structural changes and cardiac function. Blood sampling was performed in the morning after a 12-hour overnight fast. Plasma total cholesterol, triglyceride, high-density lipoprotein cholesterol (HDL-C), albumin, hemoglobin, and creatinine were measured by standard laboratory techniques. The relationships between echocardiographic parameters and various clinical characteristics, including blood pressure and laboratory data, were analyzed. This protocol was approved by the Ethics Committee of Kagawa University. Informed consent was obtained from all participants.

\section{Echocardiographic Examination}

Two-dimensional and M-mode echocardiographies were performed using the Vivid Seven System (GE; Horten, Norway). We first measured the following left ventricular structural parameters by M-mode echocardiography: ventricular septal thickness (VS) at the chordae tendineae level; left ventricular end-diastolic dimension (LVDd) and left ventricular end-systolic dimension (LVDs) at the chordae tendineae level; left ventricular posterior wall (PW) thickness at the chordae tendineae level; the end-systolic dimension of the left atrium (LAD); and the dimension of the ascending aorta (AO). The left ventricular mass was calculated according to the American Society of Echocardiography convention (Wallerson and Devereux 1987) using the following formula: left ventricular mass $=0.80[1.04$ $\left.\times(\mathrm{PW}+\mathrm{VS}+\mathrm{LVDd})^{3}-(\mathrm{LVDd})^{3}\right]+0.6$. The left ventricular mass index (LVMI) was calculated as the left ventricular mass divided by the body surface area. The left ventricular ejection fraction (LVEF) was estimated by Teichholz's method (Teichholz et al. 1976) and was used as a parameter of left ventricular systolic function. The inferior vena cava (IVC) was assessed with the patient in the supine position. Taking care to maximize the IVC diameter throughout the respiratory cycle, maximum and minimum IVC diameters $\left(\mathrm{IVC}_{\max }, \mathrm{IVC}_{\min }\right)$ were measured under normal respiration at a position $2 \mathrm{~cm}$ below the connective point between right atrium and the IVC. The respirophasic variation of IVC diameter was calculated for each patient by $\left(\mathrm{IVC}_{\max }\right.$ $\left.-\mathrm{IVC}_{\min }\right) /\left(\mathrm{IVC}_{\max }\right) \times 100$.

We next measured the parameters of LV diastolic function by recording the conventional transmitral flow velocity using pulsed Doppler echocardiography (Nishimura and Appleton 1996; Nishimura and Tajik 1997). The conventional transmitral flow velocity was recorded from the apical transducer position with the sample volume
Table 1. Clinical and Echocardiographic Characteristics of the Subjects.

\begin{tabular}{|c|c|}
\hline Number (male/female) & $200(112 / 88)$ \\
\hline $10-19$ years & 2 \\
\hline 20-29 years & 4 \\
\hline 30-39 years & 8 \\
\hline $40-49$ years & 6 \\
\hline $50-59$ years & 27 \\
\hline $60-69$ years & 56 \\
\hline 70-79 years & 60 \\
\hline $80-89$ years & 32 \\
\hline 90-99 years & 5 \\
\hline Age (years) & $66.6 \pm 14.7$ \\
\hline $\operatorname{BSA}\left(\mathrm{m}^{2}\right)$ & $1.55 \pm 0.19$ \\
\hline $\operatorname{BMI}\left(\mathrm{kg} / \mathrm{m}^{2}\right)$ & $22.1 \pm 3.5$ \\
\hline Hypertension $(n(\%))$ & $126(63)$ \\
\hline Diabetes mellitus $(n(\%))$ & $30(15)$ \\
\hline Dyslipidemia $(n(\%))$ & $59(30)$ \\
\hline Use of vasodilators $(n(\%))$ & $126(63)$ \\
\hline $\mathrm{CCB}(n(\%))$ & $68(34)$ \\
\hline ACEI/ARB $(n(\%))$ & $98(49)$ \\
\hline Systolic BP (mmHg) & $131 \pm 22$ \\
\hline Diastolic BP (mmHg) & $74 \pm 13$ \\
\hline Heart rate (beats/min) & $68 \pm 11$ \\
\hline Total cholesterol (mg/dL) & $190 \pm 35$ \\
\hline HDL cholesterol (mg/dL) & $49 \pm 16$ \\
\hline Triglycerides (mg/dL) & $115 \pm 64$ \\
\hline Albumin (g/dL) & $3.9 \pm 0.5$ \\
\hline Hemoglobin (g/dL) & $12.4 \pm 2.0$ \\
\hline Creatinine (mg/dL) & $0.73 \pm 0.21$ \\
\hline Presence of mild tricuspid regurgitation $(n(\%))$ & $82(41)$ \\
\hline \multicolumn{2}{|l|}{ Echocardiographic structural parameters } \\
\hline $\mathrm{VS}(\mathrm{mm})$ & $10.5 \pm 2.5$ \\
\hline $\mathrm{PW}(\mathrm{mm})$ & $9.8 \pm 1.8$ \\
\hline LVDd (mm) & $46.0 \pm 4.8$ \\
\hline LVDs (mm) & $27.3 \pm 4.1$ \\
\hline $\operatorname{LVMI}\left(\mathrm{g} / \mathrm{m}^{2}\right)$ & $108 \pm 30$ \\
\hline $\mathrm{LAD}(\mathrm{mm})$ & $36.2 \pm 6.1$ \\
\hline $\mathrm{AO}(\mathrm{mm})$ & $31.1 \pm 4.0$ \\
\hline \multicolumn{2}{|l|}{ Echocardiographic functional parameters } \\
\hline $\operatorname{LVEF}(\%)$ & $71 \pm 7$ \\
\hline $\mathrm{E}(\mathrm{cm} / \mathrm{s})$ & $54 \pm 15$ \\
\hline $\mathrm{A}(\mathrm{cm} / \mathrm{s})$ & $69 \pm 16$ \\
\hline $\mathrm{E} / \mathrm{A}$ & $0.82 \pm 0.33$ \\
\hline E/e' & $10.1 \pm 3.6$ \\
\hline \multicolumn{2}{|l|}{ IVC parameters } \\
\hline Maximum IVC diameter (mm) & $11 \pm 4$ \\
\hline Minimum IVC diameter (mm) & $5 \pm 3$ \\
\hline Respirophasic variation of IVC diameter (\%) & $55 \pm 19$ \\
\hline
\end{tabular}

BSA, body surface area; BMI, body mass index; CCB, calcium channel blockers; ACEI, angiotensin-converting enzyme inhibitors; ARB; angiotensin II receptor blockers; BP, blood pressure; HDL, high-density lipoprotein; VS, ventricular septal thickness; PW, left ventricular posterior wall thickness; LVDd, left ventricular end-diastolic dimension; LVDs, left ventricular end-systolic dimension; LVMI, left ventricular mass index; LAD, left atrial dimension; $\mathrm{AO}$, dimension of ascending aorta; LVEF, left ventricular ejection fraction; E, peak early diastolic transmitral flow; A, peak late diastolic transmitral flow; E/A, the ratio of E to A; e', peak early diastolic mitral annular velocity; E/e', the ratio of $\mathrm{E}$ to e'; IVC, inferior vena cava. 
Table 2. Correlation Coefficients of Linear Regression Analysis between IVC Diameters and Other Parameters.

\begin{tabular}{|c|c|c|c|c|}
\hline & \multicolumn{2}{|c|}{ Maximum IVC } & \multicolumn{2}{|c|}{ Minimum IVC } \\
\hline & $r$ & $p$ value & $r$ & $p$ value \\
\hline Age & -0.221 & 0.002 & -0.265 & $<0.001$ \\
\hline BSA & 0.238 & 0.001 & 0.215 & 0.002 \\
\hline BMI & 0.332 & $<0.001$ & 0.317 & $<0.001$ \\
\hline Hypertension & 0.022 & NS & 0.016 & NS \\
\hline Diabetes mellitus & 0.094 & NS & 0.067 & NS \\
\hline Dyslipidemia & 0.088 & NS & 0.139 & NS \\
\hline Systolic BP & 0.052 & NS & 0.107 & NS \\
\hline Diastolic BP & 0.102 & NS & 0.106 & NS \\
\hline Heart rate & -0.242 & 0.001 & -0.117 & NS \\
\hline Total cholesterol & 0.013 & NS & 0.048 & NS \\
\hline HDL cholesterol & 0.091 & NS & 0.025 & NS \\
\hline Triglycerides & 0.035 & NS & 0.037 & NS \\
\hline Albumin & 0.120 & NS & 0.080 & NS \\
\hline Hemoglobin & 0.115 & NS & 0.095 & NS \\
\hline Creatinine & -0.140 & NS & -0.104 & NS \\
\hline \multicolumn{5}{|c|}{ Echocardiographic structural parameters } \\
\hline LVDd & 0.242 & 0.001 & 0.125 & NS \\
\hline LVDs & 0.057 & NS & -0.018 & NS \\
\hline LVMI & 0.159 & 0.025 & 0.124 & NS \\
\hline LAD & 0.306 & $<0.001$ & 0.170 & 0.017 \\
\hline $\mathrm{AO}$ & -0.010 & NS & -0.023 & NS \\
\hline \multicolumn{5}{|c|}{ Echocardiographic functional parameters } \\
\hline LVEF & 0.127 & NS & 0.120 & NS \\
\hline $\mathrm{E}(\mathrm{cm} / \mathrm{s})$ & 0.345 & $<0.001$ & 0.297 & $<0.001$ \\
\hline $\mathrm{A}(\mathrm{cm} / \mathrm{s})$ & 0.016 & NS & -0.044 & NS \\
\hline $\mathrm{E} / \mathrm{A}$ & 0.215 & 0.002 & 0.205 & 0.004 \\
\hline $\mathrm{E} / \mathrm{e}^{\prime}$ & 0.120 & NS & 0.040 & NS \\
\hline
\end{tabular}

IVC, inferior vena cava; BSA, body surface area; BMI, body mass index; BP, blood pressure; HDL, highdensity lipoprotein; LVDs, left ventricular end-systolic dimension; LVDd, left ventricular end-diastolic dimension; LVMI, left ventricular mass index; LAD, left atrial dimension; AO, dimension of ascending aorta; LVEF, left ventricular ejection fraction; E, peak early diastolic transmitral flow; A, peak late diastolic transmitral flow; $\mathrm{E} / \mathrm{A}$, the ratio of $\mathrm{E}$ to $\mathrm{A}$; e', peak early diastolic mitral annular velocity; E/e', the ratio of $\mathrm{E}$ to e'.

situated between the mitral leaflet tips. The peak velocity of early transmitral flow velocity (E velocity) and the peak velocity of late transmitral flow velocity (A velocity) were recorded, and the ratio of E to A (E/A ratio) was calculated. Furthermore, tissue Doppler echocardiography (Ommen et al. 2000) was performed by activating the tissue Doppler echocardiographic function in the same machine. Mitral annular velocities were recorded from the apical 4-chamber view. Sample volumes were located at the septal site of the mitral annulus. The peak early diastolic mitral annular velocity (e') was measured, and the ratio of E velocity to e' velocity (E/e') was calculated and was used as a parameter of LV preload.

\section{Statistical Analysis}

Data are expressed as means \pm S.D. Statistical analysis was performed using the SPSS software package (SPSS, Chicago, IL). Linear regression analysis was performed to evaluate the association between IVC diameter and other variables. Stepwise multiple regression analysis was performed to determine the correlation between IVC diame- ter and each independent variable. Stepwise multiple regression analysis was performed for variables that were statistically significant in linear regression analysis. The levels for inclusion and exclusion of individual variables were 0.05 and 0.10 . Values of $p<0.05$ were considered to indicate statistical significance.

\section{Results}

Clinical and Echocardiographic Characteristics of Subjects

The clinical and echocardiographic parameters of the subjects are summarized in Table 1. The percentage $(63 \%)$ of subjects with hypertension was highest among those with cardiovascular risk factors. Including hypertensive patients in this study resulted in an above-average mean systolic blood pressure level $(131 \pm 22 \mathrm{mmHg})$. The mean LVEF was $72 \pm 7 \%$; all patients had normal systolic function (LVEF $\geq 55 \%)$. The slightly elevated mean LVMI (108 \pm $30 \mathrm{~g} / \mathrm{m}^{2}$ ) indicated the presence of LV hypertrophy in the 
Table 3. Correlation Coefficients of Linear Regression Analysis between Respirophasic Variation of IVC Diameter and Other Parameters.

\begin{tabular}{lcc}
\hline & $r$ & $p$ value \\
\hline Age & 0.244 & 0.001 \\
BSA & -0.143 & 0.043 \\
BMI & -0.187 & 0.008 \\
Hypertension & 0.057 & $\mathrm{NS}$ \\
Diabetes mellitus & -0.013 & $\mathrm{NS}$ \\
Dyslipidemia & -0.115 & $\mathrm{NS}$ \\
Systolic BP & -0.101 & $\mathrm{NS}$ \\
Diastolic BP & -0.141 & $\mathrm{NS}$ \\
Heart rate & 0.001 & $\mathrm{NS}$ \\
Total cholesterol & -0.062 & $\mathrm{NS}$ \\
HDL cholesterol & -0.007 & $\mathrm{NS}$ \\
Triglycerides & -0.024 & $\mathrm{NS}$ \\
Albumin & -0.039 & $\mathrm{NS}$ \\
Hemoglobin & -0.074 & $\mathrm{NS}$ \\
Creatinine & 0.131 & $\mathrm{NS}$ \\
Echocardiographic structural parameters & & \\
$\quad$ LVDs & 0.092 & $\mathrm{NS}$ \\
$\quad$ LVDd & 0.024 & $\mathrm{NS}$ \\
$\quad$ LVMI & 0.038 & $\mathrm{NS}$ \\
LAD & 0.074 & $\mathrm{NS}$ \\
AO & 0.010 & $\mathrm{NS}$ \\
Echocardiographic functional parameters & & \\
LVEF & -0.088 & $\mathrm{NS}$ \\
E (cm/s) & -0.186 & 0.009 \\
A (cm/s) & 0.124 & $\mathrm{NS}$ \\
E/A & -0.182 & 0.010 \\
E/e' & 0.088 & $\mathrm{NS}$ \\
\hline$\quad$ IVC, & & \\
\hline
\end{tabular}

IVC, inferior vena cava; BSA, body surface area; BMI, body mass index; BP, blood pressure; HDL, high-density lipoprotein; LVDs, left ventricular end-systolic dimension; LVDd, left ventricular end-diastolic dimension; LVMI, left ventricular mass index; LAD, left atrial dimension; AO, dimension of ascending aorta; LVEF, left ventricular ejection fraction; E, peak early diastolic transmitral flow; A, peak late diastolic transmitral flow; $\mathrm{E} / \mathrm{A}$, the ratio of $\mathrm{E}$ to $\mathrm{A}$; e', peak early diastolic mitral annular velocity; E/e', the ratio of $\mathrm{E}$ to $\mathrm{e}^{\prime}$.

subjects. In addition, the decreased mean E/A $(0.82 \pm 0.33)$ indicated LV diastolic dysfunction accompanying LV hypertrophy. However, maximum (11 $\pm 4 \mathrm{~mm})$ and minimum (5 $\pm 3 \mathrm{~mm}$ ) IVC diameters and the respirophasic variation (55 $\pm 19 \%$ ) of the IVC diameter were considered to be normal.

\section{Association between IVC Diameters and Other Variables}

Linear regression analysis was performed to examine the relationship between IVC diameters and other variables in all subjects (Tables 2 and 3). The maximum IVC diameter showed statistically significant correlations with age $(r=$ $-0.221, p=0.002)$, body surface area (BSA) $(r=0.238, p=$ $0.001)$, body mass index (BMI) $(r=0.332, p<0.001)$, heart rate $(r=-0.242, p=0.001), \operatorname{LVDd}(r=0.242, p=0.001)$, LVMI $(r=0.159, p=0.0259$, LAD $(r=0.306, p<0.001)$, $\mathrm{E}(r=0.345, p<0.001)$, and $\mathrm{E} / \mathrm{A}(r=0.215, p=0.002)$
(Table 2). The respirophasic variation of the IVC showed statistically significant correlations with age $(r=0.244, p=$ $0.001)$, BSA $(r=-0.143, p=0.043)$, BMI $(r=-0.187, p=$ $0.008), \mathrm{E}(r=-0.186, p=0.009)$, and $\mathrm{E} / \mathrm{A}(r=-0.182, p=$ 0.010) (Table 3).

\section{Assessment of the Factors Related to IVC Diameters}

Stepwise multiple regression analysis was performed to identify which clinical and echocardiographic parameters were independently associated with IVC diameters. Stepwise multiple regression analysis was performed for variables that were statistically significant in linear regression analyses (Tables 2 and 3). This analysis indicated that $\mathrm{E}(\beta$ coefficient $=0.264, p<0.001), \mathrm{LAD}(\beta$ coefficient $=$ $0.370, p<0.001)$, and age ( $($ coefficient $=-0.249, p<0.001)$ were independent determinants of maximum IVC (Table 4) 
Table 4. Multiple Regression Analysis for Maximum and Minimum IVC Diameters and Related Parameters.

\begin{tabular}{|c|c|c|c|}
\hline Independent variable & B coefficient & $t$ value & $p$ value \\
\hline $\mathrm{E}$ & 0.261 & 3.934 & $<0.001$ \\
\hline LAD & 0.370 & 5.716 & $<0.001$ \\
\hline \multirow[t]{2}{*}{ Age } & -0.249 & -3.706 & $<0.001$ \\
\hline & $\begin{array}{c}F \text { ratio }=23.028 \\
\quad(p<0.001)\end{array}$ & \multicolumn{2}{|c|}{$r^{2}=0.278$} \\
\hline \multicolumn{4}{|l|}{ Minimum IVC } \\
\hline Independent variable & $ß$ coefficient & $t$ value & $p$ value \\
\hline BMI & 0.343 & 5.137 & $<0.001$ \\
\hline $\mathrm{E} / \mathrm{A}$ & 0.292 & 4.372 & $<0.001$ \\
\hline & $\begin{array}{c}F \text { ratio }=22.428 \\
\quad(p<0.001)\end{array}$ & \multicolumn{2}{|c|}{$r^{2}=0.199$} \\
\hline
\end{tabular}

IVC, inferior vena cava, E, peak early diastolic transmitral flow; LAD, left atrial dimension; BMI, body mass index; A, peak late diastolic transmitral flow; E/A, the ratio of $\mathrm{E}$ to $\mathrm{A}$.

Table 5. Multiple Regression Analysis for Respirophasic Variation of IVC Diameter and Related Parameters.

\begin{tabular}{lccc}
\hline Independent variable & $\beta$ coefficient & $t$ value & $p$ value \\
\hline Age & 0.268 & 3.737 & $<0.001$ \\
& $\begin{array}{c}F \text { ratio }=13.967 \\
(p<0.001)\end{array}$ & $r^{2}=0.072$ & \\
\hline
\end{tabular}

IVC, inferior vena cava; BMI, body mass index.

and that age $(\beta$ coefficient $=0.268, p<0.001)$ was an independent determinant of the respirophasic variation of the IVC diameter (Table 5). Therefore, only age was an independent determinant of both maximum IVC diameter and the respirophasic variation of the IVC diameter, although the correlations were weak (Fig. 1).

\section{Discussion}

The present study presents data regarding the relationships between IVC diameters and echocardiographic and clinical parameters in patients with cardiovascular risk factors, but without overt heart diseases. The data led us to the following conclusions. First, the maximum IVC is decreased and respirophasic variation of IVC diameters is increased with advancing age. Second, among all echocardiographic parameters, LAD and $\mathrm{E}$ are associated with maximum IVC diameter.

To our knowledge, this is the first report regarding agerelated changes in IVC diameters in Japanese subjects without overt heart disease. However, all subjects in the present study had normal IVC diameters (Table 1) and were considered to have no elevated right atrial pressure. Therefore, the age-related changes in IVC diameters in the present study may not have relevance in diagnosing or treating patients with right-side heart failure. Because the parameters of IVC diameters in the present study were in the normal range, it is difficult to quantify the right atrial pressure by using these IVC parameters. However, our findings may suggest that some elderly people in the present study may have low right atrial pressure compared to younger people. The elderly patients in the present study showed decreased maximum IVC diameter and increased respirophasic variation of the IVC diameter (Fig. 1). The previous studies (Simonson and Schiller 1988; Kircher et al. 1990; Nagueh et al. 1996; Brennan et al. 2007) demonstrated that decreased IVC diameter and increased respirophasic IVC collapsibility indicated decreased right atrial pressure. Therefore, the elderly patients in the present study may have had low right atrial pressure. Another reason of the age-related changes in IVC diameter may be a decreased IVC compliance with advancing age. Even if right atrial pressure does not change with advancing age, IVC diameter would be smaller because of a decrease in IVC compliance with advancing age. These characteristics of elderly patients should be kept in mind when performing echocardiographic examinations in elderly patients.

The subjects of the present study included a lot of hypertensive patients. We compared the age-related changes in IVC parameters between 74 normotensive and 126 hypertensive patients in the present study. Although the 

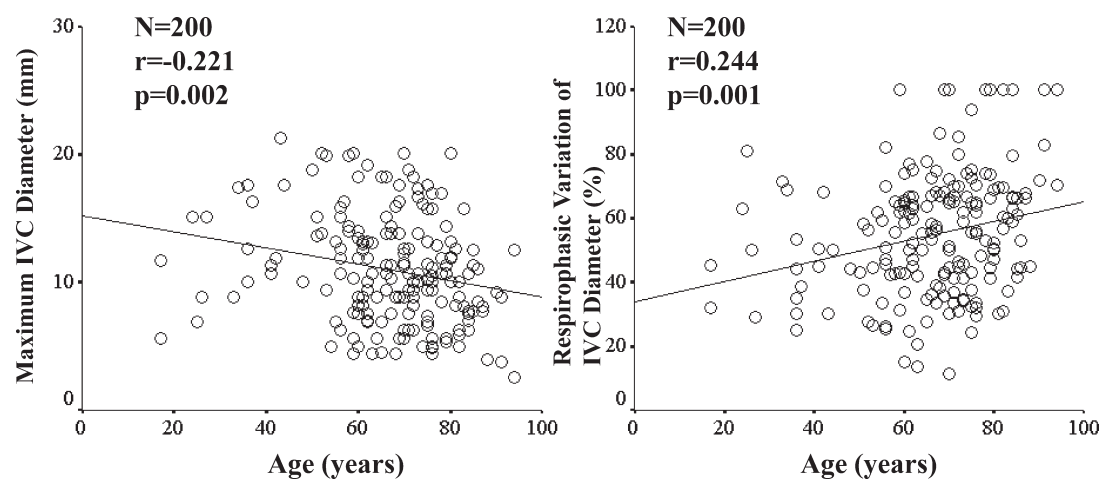

Fig. 1. Relationships between age, maximum IVC diameter, and respirophasic variation of IVC diameter. Age was correlated with both maximum IVC diameter $(r=-0.221, p=0.002)$ and respirophasic variation of IVC diameter $(r=0.244, p=0.001)$, but the correlations were weak. IVC, inferior vena cava.

maximum $(r=-0.042, p=0.724)$ and minimum $(r=-0.194$, $p=0.098)$ IVC diameters did not correlate with age in normotensive patients, the maximum $(r=-0.417, p<0.001)$ and minimum $(r=-0.364, p<0.001)$ IVC diameters correlated with age in hypertensive patients. However, the respirophasic variations of the IVC correlated with age in both normotensive $(r=0.273, p=0.019)$ and hypertensive $(r=$ $0.217, p=0.015)$ patients. Thus, the differences in the agerelated changes in IVC diameters were observed between normotensive and hypertensive patients. However, the number of patients was considerably different between the two groups. In addition, the mean age ( $70 \pm 12$ years) of hypertensive patients was significantly higher than that $(61 \pm 18$ years) of normotensive patients $(p<0.001)$. Therefore, it is difficult to determine whether the age-related changes in IVC diameter differ between normotensive and hypertensive patients. Further studies for large number of normotensive patients are needed to determine the influences of hypertension on IVC diameters.

The present study has several limitations. First, because the participants of the present study were patients with cardiovascular risk factors including hypertension, some patients may have had left ventricular hypertrophy or ischemic heart disease. Age-related changes in IVC diameter in normal subjects without cardiovascular risk factors may be different from the results of the present study. Second, because the present study included 82 patients ( $41 \%$ of all patients) with mild tricuspid regurgitation (Table 1 ), tricuspid valve regurgitation may have influenced the results. However, because many normal subjects or patients with cardiovascular risk factors have tricuspid regurgitation to some degree, it is difficult to assess IVC diameter without the effects of tricuspid valve regurgitation. Third, vasodilators, including calcium channel blockers, angiotensin-converting enzyme inhibitors, and angiotensin II receptor blockers, may have influenced on IVC diameter in the present study. We compared maximum and minimum IVC diameters between the patients with vasodilators and those without vasodilators. However, no differences in maximum (with vasodilators: $11 \pm 4$ vs. without vasodilators: $11 \pm 4$ $\mathrm{mm}$ ) and minimum (with vasodilators: $5 \pm 3$ vs. without vasodilators: $5 \pm 3 \mathrm{~mm}$ ) IVC diameters were observed between the two groups. Fourth, we had small number of younger patients in the present study (Table 1). Further studies including equal number of patients in each age stratum are needed. Finally, we used M-mode echocardiography for measuring IVC diameters in the present study. As the point where the IVC diameter was measured, moved throughout the respiratory cycle, the values of IVC diameters in the present study may be different from those obtained by 2-dimensional echocardiography. Further studies using 2-dimensional echocardiography are needed for elucidating normal values of IVC diameter.

In conclusion, maximum IVC diameter is decreased and respirophasic IVC collapsibility is increased with advancing age. The age-related changes in IVC diameter suggest that right atrial pressure may be decreased with advancing age in some patients without overt cardiac disease.

\section{References}

Brennan, J.M., Blair, J.E., Goonewardena, S., Ronan, A., Shah, D., Vasaiwala, S., Kirkpatrick, J.N. \& Spencer, K.T. (2007) Reappraisal of the use of inferior vena cava for estimating right atrial pressure. J. Am. Soc. Echocardiogr., 20, 857-861.

Daimon, M., Watanabe, H., Abe, Y., Hirata, K., Hozumi, T., Ishii, K., Ito, H., Iwakura, K., Izumi, C., Matsuzaki, M., Minagoe, S., Abe, H., Murata, K., Nakatani, S., Negishi, K., Yoshida, K., Tanabe, K., Tanaka, N., Tokai, K. \& Yoshikawa, J.; JAMP Study Investigators. (2008) Normal values of echocardiographic parameters in relation to age in a healthy Japanese population: the JAMP study. Circ. J., 72, 1859-1866.

Kircher, B.J., Himelman, R.B. \& Schiller, N.B. (1990) Noninvasive estimation of right atrial pressure from the inspiratory collapse of the inferior vena cava. Am. J. Cardiol., 66, 493-496.

Nagueh, S.F., Kopelen, H.A. \& Zoghbi, W.A. (1996) Relation of mean right atrial pressure to echocardiographic and Doppler parameters of right atrial and right ventricular function. Circulation, 93, 1160-1169.

Nishimura, R.A. \& Appleton, C.P. (1996) "Diastology": beyond E and A. J. Am. Coll. Cardiol., 27, 372-374.

Nishimura, R.A. \& Tajik, A.J. (1997) Evaluation of diastolic filling of left ventricle in health and disease: doppler echocardiography is the clinician's Rosetta Stone. J. Am. Coll. Cardiol., 30, 
8-18.

Ommen, S.R., Nishimura, R.A., Appleton, C.P., Miller, F.A., Oh, J.K., Redfield, M.M. \& Tajik, A.J. (2000) Clinical utility of Doppler echocardiography and tissue Doppler imaging in the estimation of left ventricular filling pressures. A comparative simultaneous Doppler-catheterization study. Circulation, 102, 1788-1794.

Simonson, J.S. \& Schiller, N.B. (1988) Sonospirometry: a new method for noninvasive estimation of mean right atrial pressure based on two-dimensional echographic measurements of the inferior vena cava during measured inspiration. J. Am. Coll. Cardiol., 11, 557-564.
Teichholz, L.E., Kreulen, T., Herman, M.V. \& Gorlin, R. (1976) Problems in echocardiographic volume determinations: echocardiographic-angiographic correlations in the presence of absence of asynergy. Am. J. Cardiol., 37, 7-11.

Wallerson, D.C. \& Devereux, R.B. (1987) Reproducibility of echocardiographic left ventricular measurements. Hypertension, 9(2 Pt 2), II6- II18.

Watanabe, S., Suzuki, N., Kudo, A., Suzuki, T., Abe, S., Suzuki, M., Komatsu, S., Saijo, Y. \& Murayama, N. (2005) Influence of aging on cardiac function examined by echocardiography. Tohoku J. Exp. Med., 207, 13-19. 